\title{
Integrative analysis reveals an indirect connection between COX-2/PTGS2 and extracellular matrix proteins in Ch11q-deleted neuroblastoma
}

\author{
Thatyanne Gradowski Farias da Costa do Nascimento ${ }^{1}$, Nilton de França Júnior ${ }^{1}$, Lisiane \\ de Castro Poncio ${ }^{2}$, Aline Simoneti Fonseca ${ }^{2}$, Bonald Figueiredo ${ }^{2}$, Saulo Henrique Weber ${ }^{1}$, \\ Roberto Hirochi Herai ${ }^{1}$, Lucia de Noronha ${ }^{1}$, Luciane Cavalli ${ }^{2}$, Bruno César Feltes ${ }^{3}$, and \\ Selene Esposito ${ }^{1}$ \\ ${ }^{1}$ Pontifícia Universidade Católica do Paraná \\ ${ }^{2}$ Instituto de Pesquisa Pelé Pequeno Príncipe \\ ${ }^{3}$ Universidade Federal do Rio Grande do Sul
}

May 27, 2021

\begin{abstract}
The COX-2 protein, encoded by the PTGS2 gene, is related to tumor progression in adult and pediatric cancer. In neuroblastoma (NB), COX-2 was associated with loss of heterozygosity on the long arm of chromosome 11 (Ch11q loss of heterozygosity, LOH), defining a subset of aggressive disease. The present study aimed to investigate the protein expression of COX-2 in a set of 82 pre-chemotherapy $(\mathrm{CT})$ and 20 post-CT NB specimens and its correlation with clinical and genomic data. A systems biology approach elucidated the network interaction of PTGS2 and other inflammation-related genes with those codified in the Ch11q deleted regions. The results indicated a significantly higher expression of COX-2 in post-CT samples. In addition, a significant positive correlation between the presence of aberrations in Ch11q and COX-2 levels and an indirect connection between the COX-2 gene and extracellular matrix remodeling (ECM)-related proteins were observed. Our findings suggest that deregulation of ECM proteolysis in Ch11q-deleted NB could elicit stromal alterations, triggering inflammatory responses via COX-2 overexpression, ultimately supporting NB progression.
\end{abstract}

\section{Introduction}

Neuroblastoma (NB) is the most frequent extracranial solid tumor in children, accounting for 8-10\% of all pediatric cancers ${ }^{1}$. These tumors originate from neural crest cells, which are primitive progenitors of sympathetic ganglia, and can arise anywhere along the sympathetic nervous system ${ }^{2}$. After tumor formation, it results in a spectrum of clinical diseases ranging from variably aggressive NBs to well-differentiated benign tumors (i.e., ganglioneuroma, GN) ${ }^{3}$. Metastases are diagnosed in about $50 \%$ of patients, with the bone marrow, bone, and regional lymph nodes being

the most commonly affected sites ${ }^{4}$. NB treatment includes a wide range of therapies, depending on patients' disease risk classification ${ }^{1}$. After induction and consolidation chemotherapy, approximately half of all patients reportedly develop drug resistance or suffer disease relapse after the first-line therapy ${ }^{5,6}$.

The International NB staging system (INSS) classifies NBs into different stages (1, 2A/B, 3, 4, and 4S) based on clinical criteria ${ }^{7,}{ }^{8}$. In addition, the $M Y C N$ oncogene amplification (MNA) is an independent 
poor prognostic factor significantly associated with INSS stage 4, and unfavorable histological features ${ }^{9}$. Determination of tumor cell ploidy and the identification of segmental chromosomal aberrations found most frequently in 1p, 1q, 3p, 11q, 14q, and 17p have substantially improved NB risk stratification and the choice of the most effective treatment regimens ${ }^{9}$. Specifically, LOH in chromosome 11q (Ch11q) in nonamplified$M Y C N$ (NAMN) was found to be associated with a therapy-resistant metastatic NB subgroup ${ }^{10}$, as well as with high activity of the COX/microsomal prostaglandin E synthase (mPGES)-1/PGE2 pathway ${ }^{11}$.

Prostaglandins (PGs, including PGD2, PGE2, PGF2a, and PGI2) are arachidonic acid-derived chemical mediators of the inflammatory response ${ }^{12}$. They are produced by sequential actions of cyclooxygenases $(\mathrm{COX}-1$ or COX-2) and specific synthases, exerting their effects mainly through the G-protein-coupled receptors (GPCRs), activating adenylate cyclase or phospholipase $\mathrm{C}^{12}$. Tumor cells are often characterized by aberrant COX-2 expression, resulting from transcriptional and/or post-transcriptional and epigenetic alterations 13, 14. COX-2, which is also released by cancer-associated fibroblasts (CAFs) and type-2 macrophages (M2)

${ }^{15}$, is involved in angiogenesis, tumor cell proliferation, and survival. It correlates with invasiveness and resistance to chemotherapeutic drugs in many cancer types, such as breast, lung, colon, prostate, and bladder ${ }^{16,17}$. In NBs, high COX-2/PGE2 expression levels promote malignant cell transformation and inhibit apoptosis via cAMP-mediated $\beta$-catenin stabilization, a process that may be of particular significance in NAMN cells ${ }^{18}$.

Despite the premise that the COX-2 pathway favors tumor progression, the exact extent of this association has not yet been completely understood. Network systems biology has been broadly accepted as useful tools that allow the visualization and analysis of the interaction of multiple molecular pathways, providing the uncovering of new biomarkers and their association to disease phenotypes ${ }^{19}$. In the current study, we analyzed COX-2 expression levels in NB tumor samples obtained during diagnosis and post-chemotherapy. Furthermore, we analyzed the genomic profile of tumors with Ch11q aberrations and the correlation with the genes encoding the enzymes involved in the COX/mPGES-1/PGE2 and other inflammatory pathways using a pipeline of computational systems biology tools.

\section{Material and Methods}

\section{Tumor samples}

Neuroblastic formalin-fixed paraffin-embedded (FFPE) blocks from 2004-2014 were obtained from the Pequeno Príncipe Hospital (PPH) Biobank, and the clinical data were retrospectively collected from the PPH Service of Medical Archives and Statistics (SAME). This study was approved by the PPH Ethics Committee (CEP0518-07/2007; amendment 2014), and all patients were kept anonymous. Two subgroups of NB specimens were obtained, 82 from patients that were not submitted to any treatment (pre-CT), and 20 were obtained after chemotherapy (post-CT). In eleven patients, it was possible to obtain tumor specimens pre- and post-CT. Each sample was classified according to the following criteria: (i) age at diagnosis $(<18$ or [?] 18 months); (ii) sex; (iii) Shimada classification ${ }^{20}$ (favorable or unfavorable histological features); (iv) INSS-based staging (1-4 or $4 \mathrm{~S}$ ); and (v) clinical course of the disease (alive without disease, relapsed, deceased).

\section{Tissue microarray (TMA) construction and immunohistochemical analysis}

Ten TMAs were sampled, each of them with an average of two cores ( $4 \mu \mathrm{m}$ thick; $3 \mathrm{~mm}$ diameter) from 12 cases ${ }^{21}$. The TMAs were incubated with a primary rabbit polyclonal anti-COX-2 (1:200; Spring Bioscience, USA) and secondary-horseradish peroxidase (HRP)-conjugated antibody (Spring Bioscience USA). Positive (colon cancer specimens) and negative (omitting primary antibodies) controls were run in parallel in each of the reactions. The images were obtained using a Zeiss Axioscan Slide Scanner (Jena, Germany) in high power fields (HPFs; 20x magnification), with a total area of 90,472.78 $\mu \mathrm{m}^{2}$. Immunohistochemical expression was evaluated through quantitative analyses of cytoplasmic staining images using the Image-Pro Plus ${ }^{\circledR}$ software 
(Rockville, MD, USA) and calculated as a percentage of the ratio of positive staining area per the total area $^{22}$

\section{$M Y C N$ amplification status}

$M Y C N$ oncogene copy number was assayed by FISH on the TMAs, using a direct commercial probe (Surefish 2p24 MYCN 277kb p5; Agilent Technologies Inc., Santa Clara, CA, USA). Briefly, the TMA sections were deparaffinized, dehydrated, and treated with $\mathrm{HCl}(0.2 \mathrm{~N})$, followed by proteolytic digestion with pepsin $(750$ $\mathrm{U} / \mathrm{ml}$ ) and serial ethanol dehydration. Hybridization was performed overnight at $37{ }^{\circ} \mathrm{C}$ in a humidified chamber. The slides were counterstained with $0.2 \mu$ mol DAPI in an antifade solution. Samples were blind analyzed by manual counting by two independent investigators. Digital images were obtained using a confocal microscope (NIKON Instruments Inc., Tokyo, Japan). $M Y C N$ was considered non-amplified in samples with 2-9 copies and amplified in samples with [?] 10 positive signals.

\section{Array-comparative genomic hybridization (a-CGH) analysis}

DNA copy number analysis was performed using an oligonucleotide array-CGH platform (SurePrint G3 Human CGH Microarray 8x60K; Agilent Technologies Inc., Santa Clara, CA, USA), using a previously established protocol for FFPE samples ${ }^{23,} 24$. DNA was isolated using the standard phenol-chloroform method. Reference DNA was prepared from the peripheral blood of a pool of ten healthy donors ${ }^{25}$. Equal amounts of tumor and reference genomic DNA (1-2 $\mu \mathrm{g})$ were digested and enzymatically labeled using the SureTag Complete DNA Labeling Kit (Agilent Technologies, Inc., Santa Clara, CA, USA) and hybridized to the arrays. The array data were analyzed with the Feature Extraction (FE) v.10.10 software and Agilent CytoGenomics v.3.0 software (Agilent Technologies Inc., Santa Clara, CA, USA), using the ADM-2 algorithm, threshold 6.0 , and an aberration filter with a minimum of $>3$ probes ${ }^{25}$. Copy number gains and losses were defined as previously described ${ }^{24}$. Cytobands that showed copy number alterations (CNAs) in the Ch11q-deleted locus in pre- and post-CT samples from three patients were identified using Agilent CytoGenomics v.7.0 interval base reports (Agilent Technologies, Inc., Santa Clara, CA, USA). Genes were extracted from these cytobands using the UCSC genome browser ${ }^{26}$ and the RefSeq gene model. The online data mining software BioMart of the Ensembl platform (http://m.ensembl.org/; release 101, August 2020) was used to select the protein-coding genes within this region. DIANA-miRPath v.3 and TarBase v.7.0 ${ }^{27},{ }^{28}$ were used to identify the predicted miRNA targets. Only miRNA/mRNA targets that had a TargetScan context score of -0.4 (default) were included.

\section{Systems biology analysis}

The Ch11q protein-coding genes and miRNA target genes (Supplemental Table S1) identified were used as inputs to generate a Homo sapiens protein-protein interaction (PPI) network by using the STRING database v.11.0 29. To determine interactions of COX-2/PGES1 pathway with Ch11q, PTGS2 was also included in the PPI, together with a list of other 29 inflammation-related genes obtained from two recent reviews ${ }^{17,} 30$. All active interaction sources included experiments, databases, co-expression, neighborhood, gene fusion, and co-occurrence, but not textmining. The minimum required interaction score was set at medium confidence (0.400). For topological analyzes, degree, betweenness, and eigenvector centralities were calculated. The PPI data was transferred to the Cytoscape v.3.6.1 software $^{31}$, and the CentiScape 2.2 Plug-in was used to select the centralities in the whole network. Degree measures how many direct neighbors are connected to a given node, and nodes with above-average degree values are called hubs. A bottleneck is a node with above-average betweenness, which significantly influences the network structure. Finally, the eigenvector centrality assigns a relative score to all the nodes in the network based on the concept that connections to high-scoring nodes highly contribute to the network ${ }^{31}$. High eigenvector denotes network switches in the network ${ }^{32}$. Nodes with above-average scores in all three centralities are classified as Hub-Bottlenecks-Switches (HBS) and have a key influence in regulating molecular networks.

To better understand the network organization, cluster formation was investigated with the Molecular Complex Detection (MCODE) app ${ }^{33}$. Loops, Haircut, and Fluff were chosen in the network using the advanced options. The cut-off point was delimited as many nodes $>10$ and number of connections $>3$. The Biological 
Networks Gene Ontology tool (BiNGO) plug-in was used to investigate Gene Ontologies ${ }^{34}$, with hypergeometric testing and the Bonferroni family-wise correction with a significance level p [?] 0.05. All non-specific bioprocesses, such as regulation of the biological process, regulation of the metabolic process, were excluded for further analysis considering their lack of biological meaning. The workflow of the used methods is shown in Supplemental Figure S1.

\section{Statistical analysis}

Patient sex, age-related risk, tumor stage, Shimada status, recurrence, death, and clinical follow-up data were distributed in relative frequencies. Correlation analyses among the clinical data, COX-2 protein expression, and CNAs were performed using the nonparametric Spearman multivariate test with IBM SPSS Statistics (IBM Corp., Version 23.0, Armonk, NY). For this analysis, CNAs were categorized as no alteration (0), loss or deletion $(-1)$, and gain or amplification $(+1)$. Correlations with p-values $<0.05$ were considered significant. Pre/Post-paired comparisons were performed with the Wilcoxon signed-rank test $(\mathrm{p}<0.05)$.

\section{Results \\ Patient data}

The clinical data of a total of 91 patients were analyzed in this study (TABLE 1). The patients' outcomes were examined until September 2019, 60 months after the inclusion of the last patient. The average age at diagnosis was 33.5 months, ranging from 0 to 13 years old, with 52 patients (58\%) being diagnosed after 18 months of age. Thirteen cases (14\%) were diagnosed as GN. NB tumors were classified according to the INSS ${ }^{7}$, with stage 4 as the most frequent (44\% cases), which was coincident with unfavorable histological features (48\% cases). Relapse occurred in approximately 35 cases (39\%); in total, 30 patients (33\%) died of disease, with 25 patients dying (28\%) within a 5-year follow-up period. The MNA status was determined for 84 subjects (Supplemental Figure S2). Among them, 9 cases (11\%) were classified as having amplified $M Y C N$, with > $10 M Y C N$ copies.

COX-2 expression levels were higher in post-CT tumors and inversely correlated with MYCN amplification

All the NB and GNB samples were positive for the COX-2 protein expression ( $\mathrm{n}=77$ ). COX-2 expression levels in the post-CT samples were significantly higher than the those observed in the pre-CT unpaired samples $(\mathrm{p}$-value $=0.0097 ;$ Figure 1$)$ and inversely correlated with MNA (Spearman $\rho=-0.693, \mathrm{p}$-value $=$ 0.038). In the paired samples, COX-2 expression in post-CT was slightly higher than in pre-CT ( $\mathrm{p}$-value $=$ 0.4076). In the multivariate analysis, no correlation was observed among the COX-2 expression and clinical factors. OS inversely correlated with age at diagnosis (Spearman $\rho=-0.295$, p-value $=0.009)$, stage $(\rho=$ $-0.422, \mathrm{p}<0.0001)$, and MNA status $(\rho=-0.562, \mathrm{p}<0.0001)$.

\section{Primary tumors with Ch11q deletions presented higher COX-2 expression levels}

Array-CGH analysis in the paired sample sets $(\mathrm{n}=11)$ revealed that the median (min-max) of the CNAs in each case did not significantly differ between the pre-CT [14.5 (3-47)] and post-CT [9 (2-29)] (p-value $=0.3813$ ) (Figure 2A). The most frequent aberrant cytobands (present in at least $50 \%$ of cases) were compared in the paired samples (Figure 2B). The cytobands that (i) correlated with COX-2 expression in the multivariate analysis, (ii) were within the chromosomal regions that code for COX-2/PGE2 pathway proteins, and (iii) have prognostic relevance to NBs (cytobands 2p24, 11q12-q25, and 17q11-q25) ${ }^{35}$. CNAs distribution and frequency varied randomly between pre-CT and post-CT tumor samples. Cytobands 7p22p15, 7q11-q36 (gain), and 10q11-q26 (loss) were mainly or exclusively found in the pre-CT samples. In contrast, CNAs were observed in a higher frequency after chemotherapy in the $2 \mathrm{p}(36 \%), 4 \mathrm{p}(63 \%), 14 \mathrm{q}$ $(72 \%)$, and $17 q(54 \%)$. Positive correlations between COX-2 expression levels and $7 \mathrm{p}$ gain $(\rho=0.798 ; \mathrm{p}$-value $=0.006), 7 \mathrm{q}$ gain $(\rho=0.853 ; \mathrm{p}$-value $=0.002)$, and 11q loss $(\rho=0.631, \mathrm{p}=0.045)$ were observed. These cases were then segregated into three groups based on $M Y C N$ amplification status and Ch11q deletion: amplified $M Y C N$ with normal Ch11q (MNA/11q ${ }^{\mathrm{N}}$, Group 1), non-amplified $M Y C N$ with loss in Ch11q (NAMN/11q Group 2), and non-amplified $M Y C N$ with normal Ch11q (NAMN $/ 11 \mathrm{q}^{\mathrm{N}}$, Group 3) (Table 2). In general, 
Groups 1 and 2 were classified as unfavorable NBs, although in Group 2, a higher number of CNAs and higher COX-2 expression were observed in comparison with Group 1.

Genes mapped at the genomic loci 11q13.4-q24.3 interval are associated with cell adhesion and inflammation pathways

To investigate the association between CNAs in Ch11q and COX-2 protein expression, we selected the cytobands with CNAs in the following genomic loci of three patients in Group 2: 11q13.4-q25 (Pat15 and Pat20), and 11q12.1-q25 (Pat42), with a common interval among all three patients at 11q13.4-q24.3 (Figure $3 \mathrm{~A})$. Identification of the genes mapped in the common interval revealed a total of 409 protein-coding genes and 34 miRNAs (Supplemental Tables S1, S2 and S3). Kyoto Encyclopedia of Genes and Genomes (KEGG) pathway enrichment analysis (DIANA-miRPath v.3) associated 14 of the miRNAs with glycosphingolipid, fatty acid, and steroid hormone biosynthesis and cell adhesion molecules (Supplemental Table S4). Using TarBase v.7.0 miRNA target prediction analysis, 39 targets were predicted to be regulated by these miRNAs.

The list composed of the 409 protein-coding genes, the 39 miRNA targets, and the 30 inflammation-related genes ${ }^{17,} 30$ was used to generate a PPI network. It resulted in a network with 469 nodes and 476 edges (Figure $4 \mathrm{~A}$ ), with a PPI enrichment p-value of $1.0 \mathrm{e}-16$, which indicates that proteins in the network present more mutual interactions than expected. Surprisingly, no direct connection was found betweenPTGS2 and Ch11qrelated genes. Thus, indirect relationship analysis was performed by applying the degree, betweenness, and eigenvector centrality. Ninety-two out of the 469 nodes were of more topological relevance in the network: 29 hubs, 6 bottlenecks, 14 switches, and 30 HBS (Figure 4). Besides, gene ontologies (GO) analysis revealed cell adhesion, transcription, DNA repair, and inflammation as the main biological processes related to these genes. Clustering and GOs analysis led to identifying five clusters (Figure 4B; Supplemental Table S5). Cluster 1 is associated with the regulation of cytokine production, immune system process, apoptosis, cell proliferation, programmed cell death, and the mutual interaction of metalloproteinases (MMPs), which is involved in the collagen metabolic process. Cell and biological adhesion were the only processes associated with Clusters 2 and 4 . ThePTGS2 gene is an HBS node found only in cluster 3, where its interaction with other genes of the PGE2 and other inflammatory pathways is evident, as well as its indirect interaction with $C L P B$ (chromosome location Ch11q13.4), a gene involved in the cellular heat response. In cluster 5, several genes were identified related to the neurological system process, in addition to a connection of interleukin 6 (IL-6 ), IL-6 receptor (IL-6R), and signal transducer and activator of transcription 3 (STAT3 ), involved in IL-6-mediated signaling pathway. The CLPB gene, together with PTGES3 andPTGER1, are common HBS between Clusters 3 and 5. Likewise, other HBS nodes, such as the ones involving $I \Lambda-1 \beta$ and $C E B P B$ , intersect between Clusters 1 and 3, while EP300 and STAT3are common HBS to Clusters 1, 3, and 5. Altogether, this analysis led to identifying nodes of biological relevance that interconnect clusters with different profiles of gene interactions, progressing toward understanding the correlation of COX-2 expression and aberrations in Ch11q.

\section{DISCUSSION}

COX-2 has been described as a critical element in the crosstalk between cancer and inflammation in NB and other solid tumors ${ }^{18}$. However, the mechanisms involving COX-2 in tumorigenesis and the therapeutic significance of COX-2 inhibition remain elusive, especially in pediatric tumors ${ }^{16,30,36,37}$. It is not clear, for instance, if leukotriene inhibitors would be safe and effective as adjuvant therapy, at what phase of treatment, or what molecular interactions could be affected by its inhibition. In the present work, we show that COX-2 was expressed in all the analyzed NB samples, similar to previous findings ${ }^{38}$. However, COX-2 expression levels were significantly higher in post-CT specimens when compared with samples collected before treatment. The clinical validation in a higher NB patient cohort would support non-steroidal anti-inflammatory drugs in the consolidation or maintenance of NB chemotherapy regimens. By combining clinical and genomic data with network topological analysis, we also show that higher COX-2 expression may arise from the loss of a complex dynamic of interactions between components of the inflammation pathways and protein-coding genes located on Ch11q. 
MNA and Ch11q LOH are independent poor prognostic factors of high-risk $\mathrm{NB}^{9}$, almost mutually exclusive, with similar significance for prognosis ${ }^{10}$. Ch11q LOH and NAMN were observed in three patients of this study (Group 2), presenting paired specimens, either in the pre- or post-CT tumors. Expectedly, these patients presented poor prognosis factors, such as advanced age at diagnosis and a high number of CNAs. Group 2 also showed a higher expression of COX-2 protein when compared with the other groups, regardless of the MYCN status. To comprehend the correlation of Ch11q deletions and COX-2 expression, we built a PPI network including the genes located in this deleted region, COX-2/PGE2 pathway genes, and the target genes of the miRNAs codified in the deleted 11q regions. Clustering and GO analysis identified five clusters, with genes involved in different biological processes, primarily related to cancer hallmarks ${ }^{39}$. Processes, such as regulation of apoptosis, cell proliferation, and inflammation, and the ones associated with collagen metabolism were observed in cluster 1 . Cluster 3 was associated with the prostaglandin metabolic process, while cluster 5 showed an extensive network of neurological system regulation and IL-6 signaling as a particular inflammation pathway.

The nodes classified as HBS can be considered the ones with stronger topological influence, which can be due to their multiple direct interacting partners and their control of the network information flow. The genes $I \Lambda-1 \beta, C E B P B$, EP300 , and STAT3, were identified as the HBS nodes acting as integration bridges among clusters of interest. Noteworthy, all these genes were inputs from the inflammation pathways. Although molecular connections among such elements have not been shown for NB yet, other biological models demonstrate their correlation (Supplemental Figure S3). CEBPB andEP300 genes that codify for the $\mathrm{C} / \mathrm{EBP} \beta$ and p300 proteins, respectively, are both activators of PTGS2 transcription ${ }^{40,41}$. IL-1 $\beta$ protein, generated predominantly by tumor-infiltrating macrophages within the tumor microenvironment (TME), has a more functional regulatory role in COX-2 activation, regulating the expression of MMPs ${ }^{42}$, as reported for breast ${ }^{43}$ and colorectal cancers ${ }^{44}$.

MMPs are zinc-containing endopeptidases that act on the extracellular matrix (ECM). These proteins are overexpressed in CAFs and generate ECM cleavage products, such as fibronectin and collagen, which serve as chemotactic factors for inflammatory cells ${ }^{45} . M M P-3$ is a direct transcriptional target and an essential contributor to the Wnt/ $\beta$-catenin signaling pathway ${ }^{46}$. In our PPI network, MMP3 was an HBS associated with Cluster 1. It directly interacts with other MMPs and also with CTNNB1, which in turn, is linked to inflammation related HBS (EP300, TNF, and $J U N)$. The functions of $M M P-3$ are linked toMMP-1, $M M P$ 7, $M M P-10$, and $M M P-13$, all identified in the constructed PPI network, are involved in collagen catabolic and metabolic processes ${ }^{47}$ that ultimately determine the differentiation of tumor-associated immune cells ${ }^{48}$.

In summary, the PPI network analysis shed light on the conjugation of the metalloproteases with the inflammation-related genes in an indirect mode of interaction with PTGS2 . We suggest that the deficiency in MMPs, which are essential for accurate remodeling of collagen in Ch11q deleted tumors, may generate a pro-inflammatory signaling profile involving increased activities of COX-2 and other enzymes from the PGE2 production pathway through IL-1 $\beta$ activation. It is essential to mention that the loss of Ch11q does not occur alone but in association with other chromosomal aberrations in NBs, such as the loss of chromosomes $3 \mathrm{p}$ and $4 \mathrm{p}$ and gain on chromosome $7 \mathrm{q}^{49}$; however, further studies are required due to its the association with chemoresistance. Along the same line, miRNA targets found in the Ch11q region of interest were included to build our network. Interestingly $33 \%$ of these miRNA targets are coded by chromosome 1

Although the sample size limitation, the present biological analysis of network systems strategy allowed the analysis of the interaction of multiple molecular pathways, providing novel and complementary biological insights to the NBs tumorigenesis. Once these findings are clinically validated in larger and independent well-annotated cohorts of NBs patients and experimental cellular models, these findings might be translated into clinical applications for the prognosis and treatment of patients with NB.

DISCLOSURE STATEMENT: None Conflict of Interest

\section{Acknowledgments}

The authors thank the GESR of the Lombardi Comprehensive Cancer Center (Washington DC, USA) for 
performing the array-CGH slides washes and scanning, and the Center for Advanced Technologies in Fluorescence (CTAF) at the Federal University of Paraná (Curitiba, PR, Brazil) for performing FISH confocal microscopy imaging. We also acknowledge CAPES for Dr. Bruno C. Feltes postdoctoral fellowship and Ms. Akanksha Mahajan (MSc) for the technical support with array-CGH and FISH analyses.

Ethics approval and consent to participate: The present study was approved by the Human Research Ethics Committee (HPP/PR - CEP0518-07/2007; amendment 2014) of the Pequeno Príncipe Hospital (Curitiba, PR, Brazil). The analysis of the human samples used in the present study was performed following the international and national regulations in accordance with the Declaration of Helsinki.

\section{Funding}

Scholarships to Dr. Lisiane C Poncio, Ms. Thatyanne GF do Nascimento and Mr. Nilton de F Junior were provided by Coordenação de Aperfeiçoamento de Pessoal de Nível Superior (CAPES, Brazil; Finance code 001), and the scholarship to Dr Aline S Fonseca was provided by the Conselho Nacional de Desenvolvimento Científico e Tecnológico (CNPq, Brazil). Additionally, the Genomics and Epigenomics Shared Resource (GESR) partially supported the present study (grant no. P30-CA051008).

Data Availability Statement: The data that support the findings of this study are available from the corresponding author upon reasonable request.

\section{REFERENCES}

1. Tolbert VP, Matthay KK. Neuroblastoma: clinical and biological approach to risk stratification and treatment. Cell Tissue Res . 05 2018;372(2):195-209. doi:10.1007/s00441-018-2821-2

2. Matthay KK, Maris JM, Schleiermacher G, et al. Neuroblastoma.Nat Rev Dis Primers . 11 2016;2:16078. doi:10.1038/nrdp.2016.78

3. Maguire LH, Thomas AR, Goldstein AM. Tumors of the neural crest: Common themes in development and cancer. Dev Dyn . Mar 2015;244(3):311-22. doi:10.1002/dvdy.24226

4. Morgenstern DA, London WB, Stephens D, et al. Prognostic significance of pattern and burden of metastatic disease in patients with stage 4 neuroblastoma: A study from the International Neuroblastoma Risk Group database. Eur J Cancer . 09 2016;65:1-10. doi:10.1016/j.ejca.2016.06.005

5. Moreno L, Rubie H, Varo A, et al. Outcome of children with relapsed or refractory neuroblastoma: A meta-analysis of ITCC/SIOPEN European phase II clinical trials. Pediatr Blood Cancer . 01 2017;64(1):2531. doi: $10.1002 /$ pbc. 26192

6. Zhou MJ, Doral MY, DuBois SG, Villablanca JG, Yanik GA, Matthay KK. Different outcomes for relapsed versus refractory neuroblastoma after therapy with (131)I-metaiodobenzylguanidine ((131)I-MIBG). Eur $J$ Cancer . Nov 2015;51(16):2465-72. doi:10.1016/j.ejca.2015.07.023

7. Brodeur GM, Pritchard J, Berthold F, et al. Revisions of the international criteria for neuroblastoma diagnosis, staging, and response to treatment. J Clin Oncol . Aug 1993;11(8):1466-77. doi:10.1200/JCO.1993.11.8.1466

8. Meany HJ. Non-High-Risk Neuroblastoma: Classification and Achievements in Therapy. Children (Basel) . Jan 2019;6(1)doi: https://doi.org/10.3390/children6010005

9. Aygun N. Biological and Genetic Features of Neuroblastoma and Their Clinical Importance. Curr Pediatr Rev . Jan 28 2018;14(2):73-90. doi:10.2174/1573396314666180129101627

10. Mlakar V, Jurkovic Mlakar S, Lopez G, Maris JM, Ansari M, Gumy-Pause F. 11q deletion in neuroblastoma: a review of biological and clinical implications. Mol Cancer . 06 2017;16(1):114. doi:10.1186/s12943017-0686-8 
11. Larsson K, Kock A, Idborg $\mathrm{H}$, et al. COX/mPGES-1/PGE2 pathway depicts an inflammatorydependent high-risk neuroblastoma subset. Proc Natl Acad Sci U S A . Jun 2015;112(26):8070-5. doi:10.1073/pnas.1424355112

12. Korbecki J, Baranowska-Bosiacka I, Gutowska I, Chlubek D. Cyclooxygenase pathways. Acta Biochim Pol . 2014;61(4):639-49.

13. Dixon DA, Blanco FF, Bruno A, Patrignani P. Mechanistic aspects of COX-2 expression in colorectal neoplasia. Recent Results Cancer Res . 2013;191:7-37. doi:10.1007/978-3-642-30331-9_2

14. Cebola I, Peinado MA. Epigenetic deregulation of the COX pathway in cancer. Prog Lipid Res . Oct 2012;51(4):301-13. doi:10.1016/j.plipres.2012.02.005

15. Pannunzio A, Coluccia M. Cyclooxygenase-1 (COX-1) and COX-1 Inhibitors in Cancer: A Review of Oncology and Medicinal Chemistry Literature. Pharmaceuticals (Basel). Oct 11 2018;11(4):20. doi:10.3390/ph11040101

16. Hashemi Goradel N, Najafi M, Salehi E, Farhood B, Mortezaee K. Cyclooxygenase-2 in cancer: A review. J Cell Physiol . May 2019;234(5):5683-5699. doi:10.1002/jcp.27411

17. Tong D, Liu Q, Wang LA, et al. The roles of the COX2/PGE2/EP axis in therapeutic resistance. Cancer Metastasis Rev . 09 2018;37(2-3):355-368. doi:10.1007/s10555-018-9752-y

18. Jansen SR, Holman R, Hedemann I, et al. Prostaglandin E2 promotes MYCN non-amplified neuroblastoma cell survival via $\beta$-catenin stabilization. J Cell Mol Med . Jan 2015;19(1):210-26. doi:10.1111/jcmm.12418

19. Menche J, Sharma A, Kitsak M, et al. Disease networks. Uncovering disease-disease relationships through the incomplete interactome.Science . Feb 2015;347(6224):1257601. doi:10.1126/science.1257601

20. Shimada H, Ambros IM, Dehner LP, Hata J, Joshi VV, Roald B. Terminology and morphologic criteria of neuroblastic tumors: recommendations by the International Neuroblastoma Pathology Committee. Cancer . Jul 15 1999;86(2):349-63.

21. do Carmo Debur M, Raboni SM, Flizikowski FB, et al. Immunohistochemical assessment of respiratory viruses in necropsy samples from lethal non-pandemic seasonal respiratory infections. $J$ Clin Pathol . Oct 2010;63(10):930-4. doi:10.1136/jcp.2010.077867

22. Vaz de Paula CB, de Azevedo MLV, Nagashima S, et al. IL-4/IL-13 remodeling pathway of COVID-19 lung injury. Scientific reports . Oct 29 2020;10(1):18689. doi:10.1038/s41598-020-75659-5

23. Santos SC, Cavalli IJ, Ribeiro EM, et al. Patterns of DNA copy number changes in sentinel lymph node breast cancer metastases. Cytogenet Genome Res . 2008;122(1):16-21. doi:10.1159/000151311

24. Torresan C, Oliveira MM, Pereira SR, et al. Increased copy number of the DLX4 homeobox gene in breast axillary lymph node metastasis.Cancer Genet . May 2014;207(5):177-87. doi:10.1016/j.cancergen.2014.04.007

25. Sugita B, Gill M, Mahajan A, et al. Differentially expressed miRNAs in triple negative breast cancer between African-American and non-Hispanic white women. Oncotarget . Nov 2016;7(48):79274-79291. doi:10.18632/oncotarget.13024

26. Haeussler M, Zweig AS, Tyner C, et al. The UCSC Genome Browser database: 2019 update. Nucleic Acids Res . Jan 8 2019;47(D1):D853-D858. doi:10.1093/nar/gky1095

27. Vlachos IS, Kostoulas N, Vergoulis T, et al. DIANA miRPath v.2.0: investigating the combinatorial effect of microRNAs in pathways.Nucleic Acids Res. Jul 2012;40(Web Server issue):W498-504. doi:10.1093/nar/gks494 
28. Vlachos IS, Paraskevopoulou MD, Karagkouni D, et al. DIANA-TarBase v7.0: indexing more than half a million experimentally supported miRNA:mRNA interactions. Nucleic Acids Res . Jan 2015;43(Database issue):D153-9. doi:10.1093/nar/gku1215

29. Szklarczyk D, Gable AL, Lyon D, et al. STRING v11: protein-protein association networks with increased coverage, supporting functional discovery in genome-wide experimental datasets. Nucleic Acids Res. Jan 8 2019;47(D1):D607-D613. doi:10.1093/nar/gky1131

30. Qu X, Tang Y, Hua S. Immunological Approaches Towards Cancer and Inflammation: A Cross Talk. Front Immunol . 2018;9:563. doi:10.3389/fimmu.2018.00563

31. Scardoni G, Petterlini M, Laudanna C. Analyzing biological network parameters with CentiScaPe. Bioinformatics . Nov 2009;25(21):2857-9. doi:10.1093/bioinformatics/btp517

32. Feltes BC, Poloni JF, Nunes IJG, Faria SS, Dorn M. Multi-Approach Bioinformatics Analysis of Curated Omics Data Provides a Gene Expression Panorama for Multiple Cancer Types. Front Genet . 2020;11:586602. doi:10.3389/fgene.2020.586602

33. Bader GD, Hogue CW. An automated method for finding molecular complexes in large protein interaction networks. BMC Bioinformatics. Jan 2003;4:2. doi:10.1186/1471-2105-4-2

34. Maere S, Heymans K, Kuiper M. BiNGO: a Cytoscape plugin to assess overrepresentation of gene ontology categories in biological networks.Bioinformatics . Aug 2005;21(16):3448-9. doi:10.1093/bioinformatics/bti551

35. Costa RA, Seuánez HN. Investigation of major genetic alterations in neuroblastoma. Mol Biol Rep . Jun 2018;45(3):287-295. doi:10.1007/s11033-018-4161-4

36. Carlson LM, Kogner P. Neuroblastoma-related inflammation: May small doses of aspirin be suitable for small cancer patients? Oncoimmunology . Jul 2013;2(7):e24658. doi:10.4161/onci.24658

37. Comunanza V. Targeting mPGES-1 as a New Strategy against Neuroblastoma. EBioMedicine . 07 2018;33:14-15. doi:10.1016/j.ebiom.2018.06.008

38. Johnsen JI, Lindskog M, Ponthan F, et al. Cyclooxygenase-2 is expressed in neuroblastoma, and nonsteroidal anti-inflammatory drugs induce apoptosis and inhibit tumor growth in vivo. Cancer Res . Oct 2004;64(20):7210-5. doi:10.1158/0008-5472.CAN-04-1795

39. Hanahan D, Weinberg RA. Hallmarks of cancer: the next generation. Cell . Mar 2011;144(5):646-74. doi:10.1016/j.cell.2011.02.013

40. Attar N, Kurdistani SK. Exploitation of EP300 and CREBBP Lysine Acetyltransferases by Cancer. Cold Spring Harb Perspect Med . Mar 1 2017;7(3):15. doi:10.1101/cshperspect.a026534

41. Yazawa T, Imamichi Y, Yuhki KI, et al. Cyclooxygenase-2 is acutely induced by CCAAT/enhancerbinding protein beta to produce prostaglandin E 2 and F 2alpha following gonadotropin stimulation in Leydig cells.Mol Reprod Dev . Jul 2019;86(7):786-797. doi:10.1002/mrd.23163

42. Wang Y, He G, Tang H, et al. Aspirin inhibits inflammation and scar formation in the injury tendon healing through regulating JNK/STAT-3 signalling pathway. Cell Prolif . Jul 2019;52(4):e12650. doi: $10.1111 /$ cpr. 12650

43. Chung ST, Geerts D, Roseman K, Renaud A, Connelly L. Osteoprotegerin mediates tumor-promoting effects of Interleukin-1beta in breast cancer cells. Mol Cancer . Feb 1 2017;16(1):27. doi:10.1186/s12943-0170606-y

44. Kalmar A, Wichmann B, Galamb O, et al. Gene-expression analysis of a colorectal cancer-specific discriminatory transcript set on formalin-fixed, paraffin-embedded (FFPE) tissue samples.Diagnostic pathology . Jul 25 2015;10:126. doi:10.1186/s13000-015-0363-4 
45. Garner EF, Beierle EA. Cancer Stem Cells and Their Interaction with the Tumor Microenvironment in Neuroblastoma. Cancers (Basel) . Dec 2015;8(1)doi:10.3390/cancers8010005

46. Carter BZ, Mak PY, Wang X, et al. An ARC-Regulated IL1beta/Cox-2/PGE2/beta-Catenin/ARC Circuit Controls Leukemia-Microenvironment Interactions and Confers Drug Resistance in AML. Cancer Res. Mar 15 2019;79(6):1165-1177. doi:10.1158/0008-5472.CAN-18-0921

47. Fang M, Yuan J, Peng C, Li Y. Collagen as a double-edged sword in tumor progression. Tumour biology : the journal of the International Society for Oncodevelopmental Biology and Medicine. Apr 2014;35(4):287182. doi:10.1007/s13277-013-1511-7

48. Chanmee T, Ontong P, Konno K, Itano N. Tumor-associated macrophages as major players in the tumor microenvironment. Cancers (Basel) . Aug 13 2014;6(3):1670-90. doi:10.3390/cancers6031670

49. Buckley PG, Alcock L, Bryan K, et al. Chromosomal and microRNA expression patterns reveal biologically distinct subgroups of 11q- neuroblastoma. Clin Cancer Res . Jun 2010;16(11):2971-8. doi:10.1158/10780432.CCR-09-3215

\section{Tables captions}

TABLE 1 Demographic and clinical data obtained from the records of patients $(\mathrm{n}=90)$ diagnosed with ganglioneuroma (GN), neuroblastoma (NB), or ganglioneuroblastoma (GNB) during 2001-2014 at Pequeno Príncipe Hospital (Curitiba, PR, Brazil)

TABLE 2 Clinical data of patients with paired samples. The cases were classified into three groups based on the amplification status of the oncogene $M Y C N$ associated with the presence of CNAs in Chr11q cytobands. Notes: age Dx, age at diagnosis; INSS, International neuroblastoma staging system; DOD, dead of disease; NED, no evidence of disease

\section{Figures captions}

Figure 1 IHC analysis of COX-2 expression in NB tumors. Mean percentage of the stained area of COX-2 in NB/GNB. A) Unpaired $(\mathrm{n}=77)$ pre/post-CT samples. Statistical differences between pre/post groups were determined by t-test, using GraphPad Prism v.8. B) Representative IHC image of COX-2 expression in a pre- and post-chemotherapy paired sample (Patient 42; 20X magnification)

Figure 2 Cytobands with copy number alterations (CNA) in paired NB samples pre-and post-chemotherapy. A) Median of CNAs per case. B) List of CNAs affecting at least five (50\%) cases, including those of prognostic importance $(*)$ and those involved in the COX-2 pathway or correlated with COX-2 expression (\#), in preand post-treatment samples from each patient. Light grey squares indicate losses, and dark grey squares, gains. C) Prostaglandin $2\left(\mathrm{PGE}_{2}\right)$ pathway showing the main enzymes and the chromosome location of the genes involved

Figure 3 Interval of loss or deletions in Ch11q from three patients' samples classified as Group 2 $\left(\mathrm{NAMN} / 11 \mathrm{q}^{-}\right)$. A) Start and stop positions of the affected cytobands. Below: Chromosome intervals affected by CNAs for each patient in Group 2 B) Venn diagram presenting the relationships of genes located at the cytobands affected by the copy number alterations

Figure 4 PPI network topological analysis. A) Left, complete network showing the 468 gene interconnections. Nodes in blue are from the Chr11q protein-coding genes region, in yellow genes from inflammation pathways, and green Chr11q-miRNAs targets. The PTGS2gene is marked in purple. Right, Venn diagram representing CentiScape centrality analysis (degree, betweenness, eigenvector). Below, biological processes (gene ontologies - BinGo), with the highlights of five of them: steroids, cell adhesion, transcription, DNA repair, and inflammation. The adhesion process was present in all the categories. B) Clustering analysis by MCode showing the five clusters identified. Clusters 1, 3, and 5 presented several genes in common (arrows); cluster 3 was the only one composed of the PTGS2 gene. Genes marked with * are HBS genes. The common genes for Clusters 1, 3, and 5 are in the center 


\section{Supplemental Material}

Figure S1 The workflow of the methods of this study: (1) selection of samples and collection of patients' clinical data; (2) Analysis of COX-2 expression in FFPE samples; (3) genome analysis by a-CGH; (4) gene data extraction; (5) systems biology approach

Figure S2 $M Y C N$ amplification status, as determined by FISH analysis. A) Representative images of FISH analysis of NB samples hybridized with the $M Y C N$ probe. Samples classified as in (a) and (b) were considered as non-amplified $M Y C N$ (NAMN). Samples with more than ten copies were considered as amplified $M Y C N$ $(\mathrm{AMN})$. B) Percentage of NB cases $(\mathrm{n}=84)$ in each $M Y C N$ amplification group

Figure S3 Representation of the interconnection of genes of higher relevance in the network as described previously. It shows the mutual association of inflammation genes and COX-2 resulting in the activation of metalloproteinase-3

Table S1 Chr11q-deleted locus in pre- and post-CT samples from three patients

Table S2 RNA genes in Chr11q deleted cytobands.

Table S3 miRNAs pathways and targets.

Table S4 Network Analysis. Chr11q protein-coding genes, inflammation-related genes, and miRNA target genes; CentiScape Analysis; MCODE Analysis; Biological Process.

Table S5 Gene Ontologies (GO) Analysis.

\section{Hosted file}

Table_1_-_Patients.docx available at https://authorea.com/users/416158/articles/ 523819-integrative-analysis-reveals-an-indirect-connection-between-cox-2-ptgs2-andextracellular-matrix-proteins-in-ch11q-deleted-neuroblastoma

\section{Hosted file}

Table_2_Clinical_data_of_patients_with_paired_samples.docx available at https://authorea. com/users/416158/articles/523819-integrative-analysis-reveals-an-indirect-connectionbetween-cox-2-ptgs2-and-extracellular-matrix-proteins-in-ch11q-deleted-neuroblastoma 

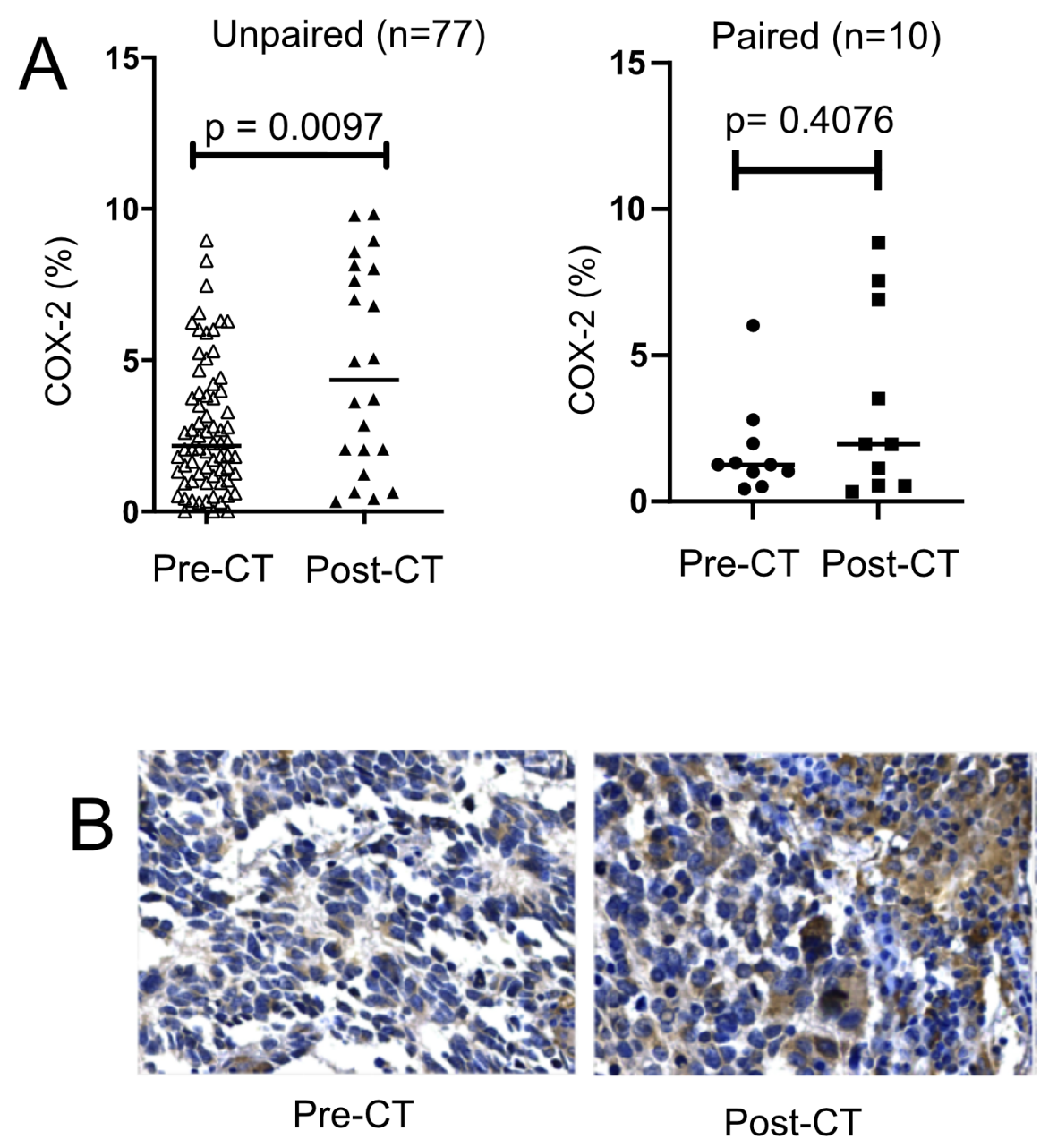
A

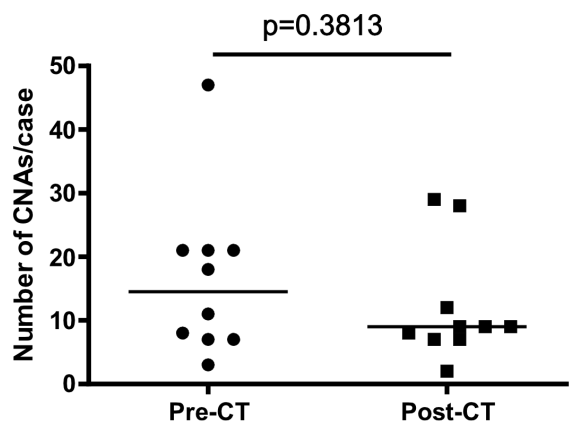

B

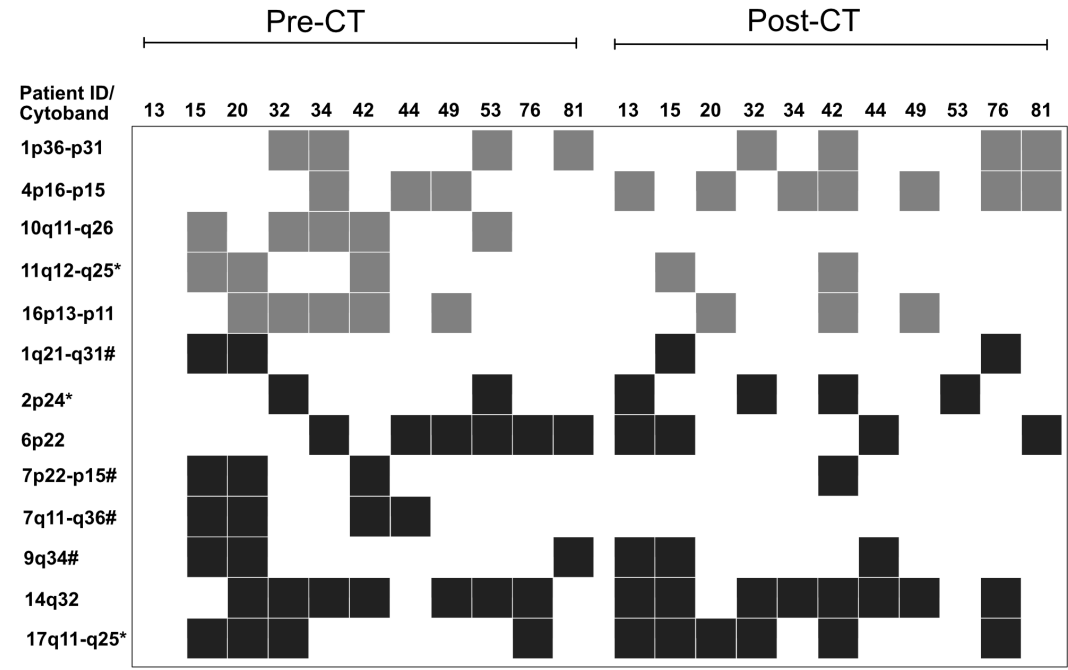

C

COX-1

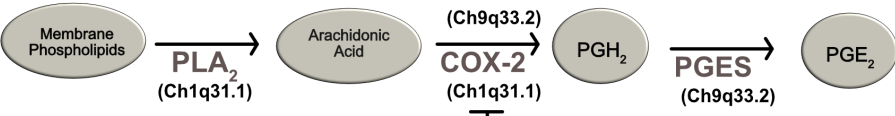

(NSAID 

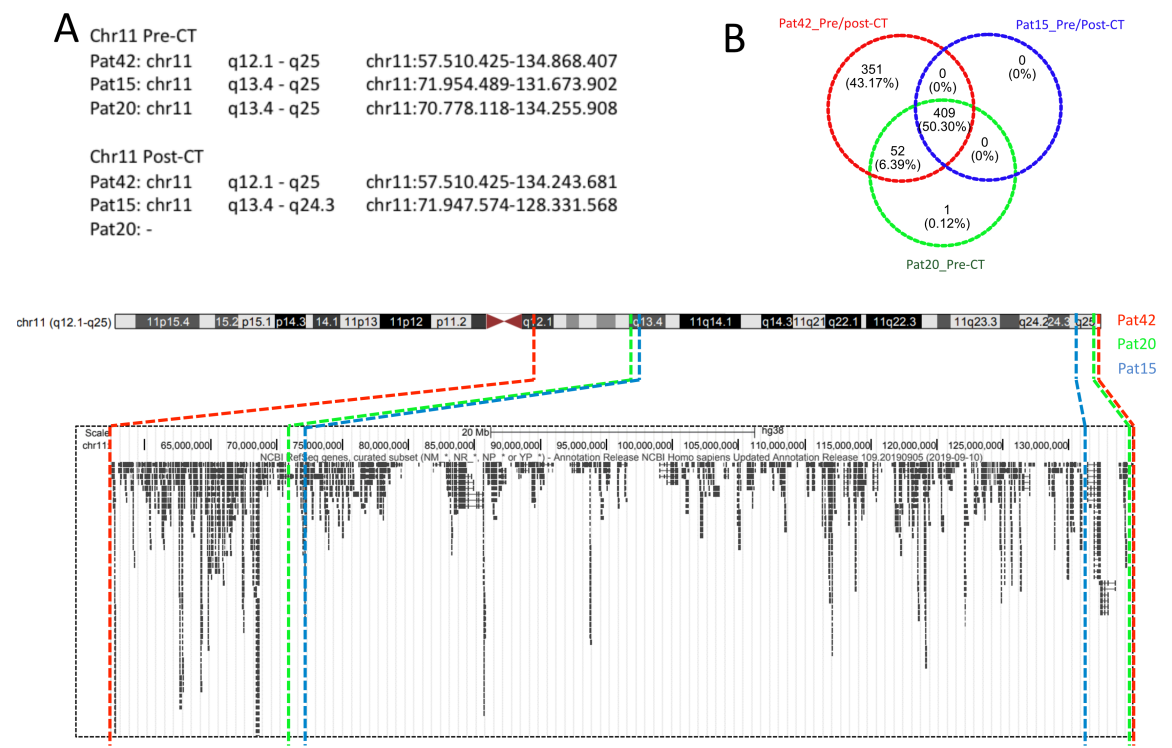

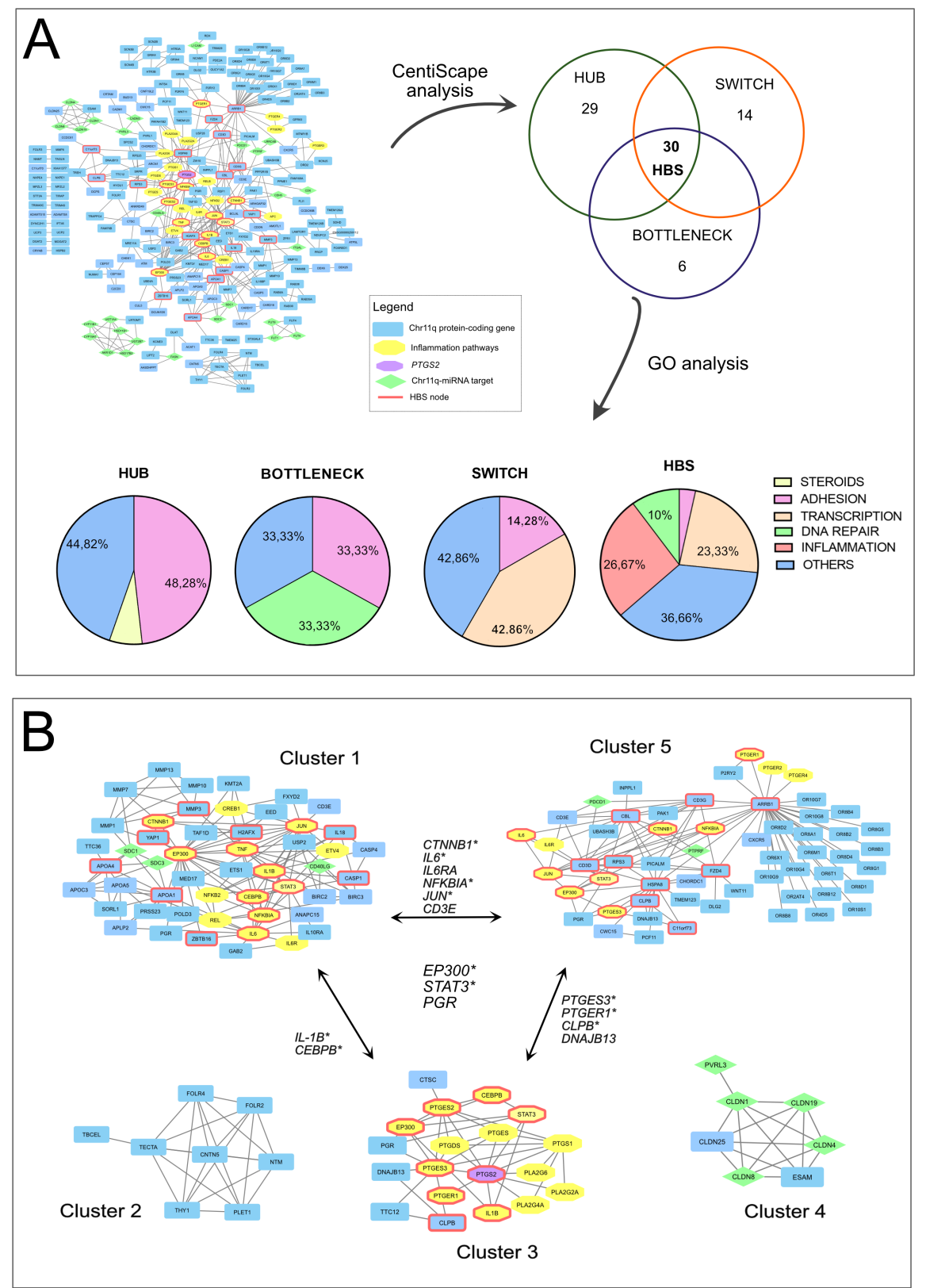